\title{
IFS and IR Observations of Star Clusters in the Antennae
}

\author{
Sabine Mengel \\ Leiden Observatory, P.O. Box 9513, NL-2300 RA Leiden, The \\ Netherlands \\ Matthew D. Lehnert, Niranjan Thatte, and Reinhard Genzel \\ Max-Planck-Institut für extraterrestrische Physik, Garching, Germany
}

\begin{abstract}
Over the past decade, it has become clear that interaction induced formation of compact young star clusters is a ubiquitous pheonomenon, and the understanding of this process is thought to also shed light on galaxy evolution in general, because these young clusters are widely believed to be the progenitors of a part of the globular cluster systems seen in local elliptical galaxies. We have observed the prototypical merger NGC 4038/4039 using near-infrared broad- and narrow band imaging, integral field spectroscopy and medium and high resolution spectroscopy. We find that all of the bright star clusters are young (<20 Myrs), but the "overlap region" hosts the youngest clusters $(\sim 5$ Myrs), while the nuclear starbursts started $\sim 100$ Myrs ago. Photometric and dynamical masses range from $10^{5}$ to a few $\times 10^{6} \mathrm{M}_{\odot}$. However, mass-to-light ratios vary from cluster to cluster and suggest differences in the contribution of low-mass stars. While clusters with a deficiency in low-mass stars are likely to evaporate before they are a Hubble time old, those with a high mass-to-light-ratio could represent young globulars.
\end{abstract}

\section{Introduction}

As has become obvious over the last years, star formation which usually accompanies violent interactions between galaxies, often occurs in the form of star cluster formation (Holtzman et al., 1992, Whitmore et al., 1993, Whitmore et al., 1999 (hereafter W99)). A common hypothesis suggests that ellipticals formed as a result of a gas-rich merger, part of their globular cluster system being evolved remnants of the young clusters. However, some aspects of this hypothesis require further investigation. For example, globular clusters have typical masses of $2 \times 10^{5} \mathrm{M}_{\odot}$ and a mass function which is lognormal. The cluster system in the Antennae, however, has a power law luminosity function (W99), and the same shape is also suggested for the mass function (Zhang and Fall, 1999). This difference in shapes of the cluster mass functions for young and globular clusters requires preferential destruction of low mass star clusters. The destruction probability depends, amongst other parameters, on the initial mass function (IMF) within the star cluster. A deficiency in low-mass stars will lead to an early disruption of the cluster (Chernoff and Weinberg, 1990). 

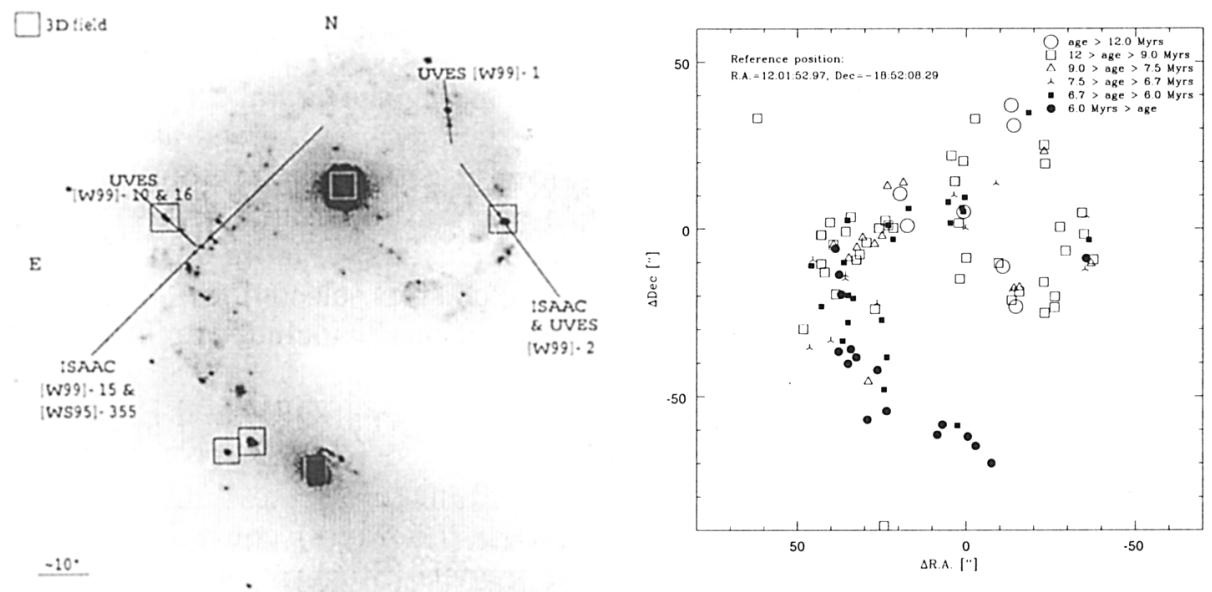

Figure 1. 1a (left): Ks-band image of NGC 4038/4039, obtained with SOFI on the NTT. Overlaid are indicators of the positions observed with MPE-3D (squares) and in spectroscopy (lines, slit length 12" with UVES and 120" with ISAAC). 1b (right): Different symbols represent different ages of star clusters detected in the SOFI Ks-band image. All of the star clusters detected here are young ( $<20$ Myrs), with the youngest clusters located in the off-nuclear overlap region.

Our observations in the near infrared (NIR) focused on the determination of the four parameters age, extinction, photometric, and dynamical cluster mass. A comparison of the latter two allows a qualitative statement about the content of low-mass stars. We used imaging (narrow and broad band), integral field spectroscopy and medium-to high resolution spectroscopy to derive these parameters.

\section{Observations, Analysis and Results}

NIR imaging of the Antennae was performed using SOFI on the NTT. The goal of these observations was an age dating and an extinction map of all the bright clusters in the merger. We selected broad band filters $\mathrm{J}, \mathrm{H}$ and $\mathrm{Ks}$, and the $\mathrm{Br} \gamma$ and $\mathrm{CO}(2.32 \mu \mathrm{m})$ narrow band filters. The field of view covered both galaxies, excluding the tails, and the FWHM of the PSF of the combined images was around $1^{\prime \prime}$.

NIR integral field spectroscopy was performed using MPE-3D on the AAT. These data sets allow to study the star formation history on small spatial scales in more detail, because each spatial pixel is covered by a spectrum with a resolution of $(\mathrm{R} \approx 1000)$. Six fields $\left(66^{\prime \prime} 4 \times 66^{\prime \prime} 4\right)$ were observed in K-band: the two nuclei and four star clusters in different regions of the merger. Data cubes were created, with $64 \times 64(0.2 /$ pix $)$ spatial and 600 spectral pixels. 
ISAAC and UVES provided the fairly high spectral resolution required for the determination of velocity dispersions. Two different slit positions including three young star clusters were observed with ISAAC on VLT-UT1 at R $\approx 9000$. The central wavelength was set to $2.31 \mu \mathrm{m}$, including the CO absorption bandheads and some continuum. Several red supergiants were observed as template stars. UVES echelle spectroscopy mainly aimed at getting high resolution spec$\operatorname{tra}(\mathrm{R} \approx 38,000)$ of the Calcium triplet absorption feature around $8500 \AA$. Three fields were observed, including a total of four clusters. Figure 1a shows the Ks-band image of NGC 4038/39, indicating the clusters selected for 3D, ISAAC and UVES observations. For our analysis, we additionally included archival HST data, and obtained the following parameters:

The extinction $\mathbf{A}_{V}$ using the comparison of $\mathrm{Br} \gamma$ (SOFI) and $\mathrm{H} \alpha$ (HST) emission and relating it to the theoretically expected (case $\mathrm{B}$ recombination) ratio. Observed values were $0.2 \mathrm{mag} \leq \mathrm{A}_{V} \leq 5.0 \mathrm{mag}$.

Ages from $\mathrm{Br} \gamma(<7.5 \mathrm{Myrs})$ and $\mathrm{CO}$ ( $>7.5 \mathrm{Myrs})$ equivalent widths The comparison with evolutionary synthesis models (Starburst99, Leitherer et al., 1999) determines the assigned age range. Starburst parameters we usually assumed are: instantaneous burst, Salpeter IMF between 1 and $100 \mathrm{M}_{\odot}, \mathrm{Z}_{\odot}$ ([W99]-2: $\left.\mathrm{Z}=2 \times \mathrm{Z}_{\odot}\right)$. The ages of all the clusters detected in the SOFI images $(\approx 150)$ are below 20 Myrs. This is very remarkable, since evidence of enhanced star formation dating back to last pericenter ( $\sim 200$ Myrs ago) could have been expected (Mihos and Hernquist, 1996). In the nuclei, the starbursts indeed started roughly 100 Myrs ago. The region where the two disks overlap hosts several very extincted star clusters, and it is the site of the most recent star formation, as was suggested by ISO data (Vigroux et al., 1996). Most of the star clusters there are around 5 Myrs old, whereas the loop located to the northwest of the merger has an average cluster age of $\sim 10$ Myrs. The $\sim 100 \mathrm{Myr}$ old clusters seen by Whitmore et al. (1999) are too faint to be identified in our NIR observations. However, star formation activity must have been much weaker at that time, otherwise many more clusters of that age would be seen now. Figure $1 \mathrm{~b}$ displays the cluster age distribution. Our determination of the cluster ages shows that the triggering of the burst cannot arise from shocks driven through the galaxies as a result of the collision during pericenter, because regions of projected distances of several kpc host essentially coeval star clusters. More plausibly, regions which had already been on the verge of collapse, like preexisting spiral arms, were driven into collapse by the changes in the tidal field during the interaction. On small spatial scales (few $100 \mathrm{pc}$ ), age differences between neighbouring clusters are observed which might be caused by direct interaction, like star formation triggered by supernovae. This is also true for the nuclei, where young clusters are seen on the background of an older starburst (Mengel et al., 2001).

Photometric masses from the K-band magnitudes in combination with the model age and the expected absolute magnitude and extinction. The masses ranged between $10^{5}$ and a few $\times 10^{6} \mathrm{M}_{\odot}$.

Dynamical masses from the line-of-sight velocity dispersion $\sigma$ and the projected half light radius $\mathrm{r}_{\mathrm{hp}}: \mathrm{M}_{\mathrm{dyn}} \approx 10 \sigma^{2} r_{\mathrm{hp}} / \mathrm{G}$. Since the cluster spectrum at an age of $\sim 10^{7}$ yrs is a superposition of red supergiants (M2I-M3I) and hot main sequence stars $(\sim \mathrm{B} 2 \mathrm{~V})$, a template spectrum is a combination of both. The 

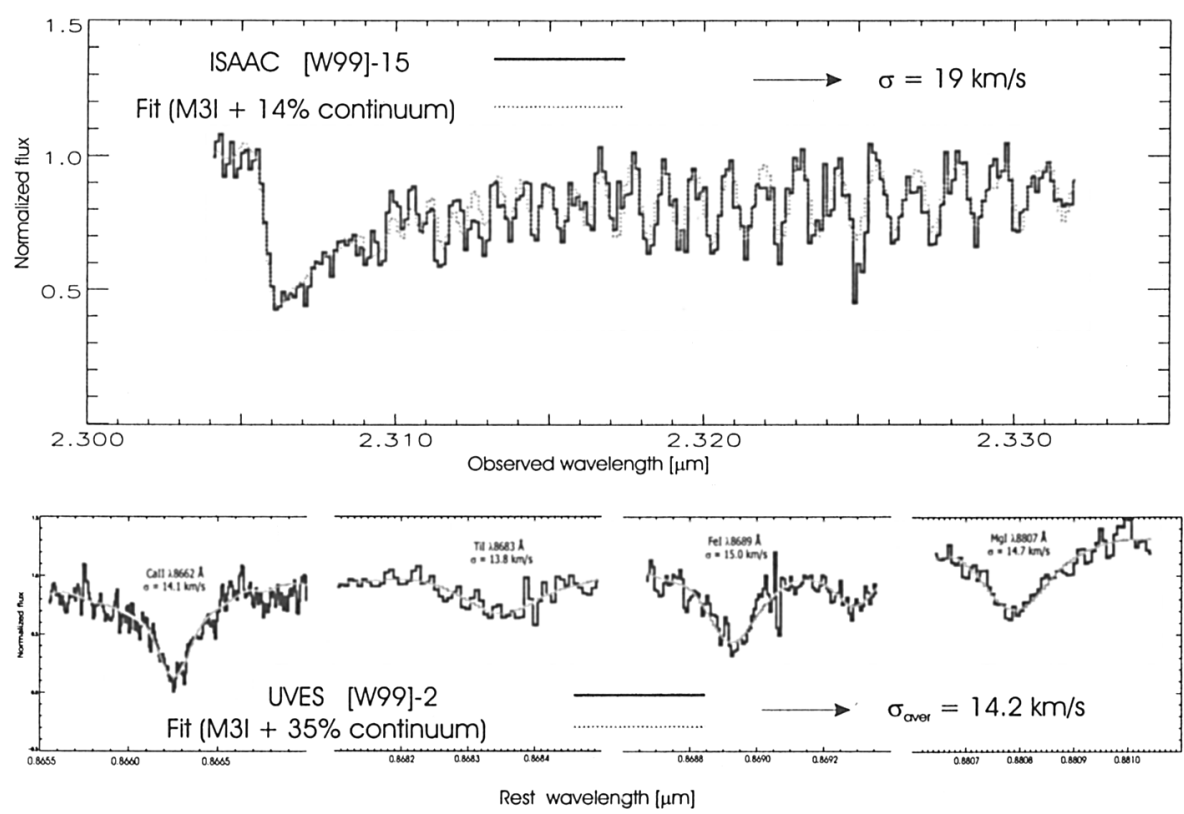

Figure 2. ISAAC ([W99]-15) and UVES ([W99]-2) spectra of two different clusters with their fits.

velocity dispersion in the cluster spectra determined from comparison with these template spectra (via $\chi^{2}(\sigma)$-minimization) is sensitive to the selected template. We established two criteria for the best matching template: 1 . The determined velocity dispersion $\sigma$ needs to be relatively independent of the fit wavelength range within the $\mathrm{CO}$ bandheads, and 2 . The rising edge of the $2.29 \mu \mathrm{m}$ bandhead reacts very sensitively to template mismatch and is required to be fit well. One of the ISAAC cluster spectra with the best fit is displayed in Figure 2 (top).

The above comments in principle also apply to the UVES spectra. Several stellar absorption lines, like the reddest line of the Calcium triplet at a rest wavelength of $8662 \AA$ and three other weaker absorption lines, were selected for the fit as it is shown in Figure 2 (bottom).

Another critical parameter concerning the mass determination of the clusters is their projected half light radius $\mathrm{r}_{\mathrm{hp}}$. We used the ishape routine developed by Larsen (1999) to perform this task. For our slightly resolved clusters, highly concentrated King profiles provided the best fit. For one cluster ([WS95]-355) we could not achieve a reliable fit and instead use the value kindly provided by Whitmore (2000, private communication). Table 1 summarizes our results for the mass determinations.

It is obvious from the table that there are vast differences in the ratios of the two mass estimates between the four clusters if the same starburst parameters (with the exception of [W99]-2: $\mathrm{Z}=2 \times \mathrm{Z}_{\odot}$ ) are assumed for all of them. In all 


\begin{tabular}{|c|c|c|c|c|c|c|c|}
\hline $\begin{array}{l}\text { Cluster } \\
\text { W99-\# }\end{array}$ & $\begin{array}{c}\text { Age } \\
{\left[10^{6} \mathrm{yr}\right]}\end{array}$ & $\begin{array}{c}\mathrm{r}_{h p} \\
{[\mathrm{pc}]}\end{array}$ & $\begin{array}{c}\mathrm{M}_{K_{0}} \\
{[\mathrm{mag}]}\end{array}$ & $\begin{array}{c}\sigma \\
{[\mathrm{km} / \mathrm{s}]}\end{array}$ & $\begin{array}{c}\mathrm{M}_{\mathrm{dyn}} \\
{\left[10^{6} \mathrm{M}_{\odot}\right]}\end{array}$ & $\begin{array}{c}\mathrm{M}_{\text {phot }} \\
{\left[10^{6} \mathrm{M}_{\odot}\right]}\end{array}$ & $\frac{M_{\text {vir }}}{M_{\text {phot }}}$ \\
\hline $355^{*}$ & $8.5^{ \pm 0.3}$ & $4.8^{ \pm 0.5}$ & $-16.1^{ \pm 0.2}$ & $20.6^{ \pm 1.1}$ & $4.7^{ \pm 1.0}$ & $0.25^{ \pm 0.25}$ & 20 \\
\hline 15 & $8.7^{ \pm 0.3}$ & $3.6^{ \pm 0.4}$ & $-15.5^{ \pm 0.2}$ & $19.0^{ \pm 1.0}$ & $3.0^{ \pm 0.6}$ & $0.15^{ \pm 0.15}$ & 20 \\
\hline 2 & $6.6^{ \pm 0.3}$ & $4.6^{ \pm 0.4}$ & $-17.5^{ \pm 0.2}$ & $13.3^{ \pm 0.5}$ & $1.9^{ \pm 0.3}$ & $1.9^{ \pm 0.3}$ & 1.0 \\
\hline 1 & $8.1^{ \pm 0.5}$ & $3.6^{ \pm 0.4}$ & $-16.5^{ \pm 0.2}$ & $9.1^{ \pm 0.8}$ & $0.69^{ \pm 0.19}$ & $0.34^{ \pm 0.15}$ & 2.0 \\
\hline
\end{tabular}

Table 1. Parameters for four of the Antennae star clusters. See the text for details on their determination. *: [WS95] number

cases, $M_{\text {vir }}$ is at least as high as $M_{\text {phot }}$, in three cases even higher. If $M_{\text {phot }}$ equals $\mathrm{M}_{\text {vir }}$ ([W99]-2), a lack of low-mass stars is indicated, because our assumed model had a lower mass cutoff at $1 \mathrm{M}_{\odot}$. Either there are actually no stars below that mass present, or the IMF slope, which we assumed to be Salpeter $(\alpha=2.35)$, is in reality flatter than that, or a combination of both. In those cases where the observed ratio is larger than 1 , the additional observed dynamical mass allows to extrapolate the assumed IMF to masses below $1 \mathrm{M}_{\odot}$ (a factor of 2.6 would correspond to an extrapolation to $0.1 \mathrm{M}_{\odot}$ with Salpeter slope), or to steepen the IMF. For example, the results for [WS95]-355 and [W99]-15 would be compatible with an IMF with an exponent of $\alpha=3.3$, and a lower mass cutoff of $0.1 \mathrm{M}_{\odot}$. Even though the uncertainties involved in the measurements are fairly substantial, there is an indication for IMF variations between different star clusters in NGC 4038/4039.

\section{References}

Chernoff D.F., \& Weinberg M.D. 1990, ApJ, 351, 121

Díaz A.I., Terlevich E., \& Terlevich R. 1989, MNRAS 239, 325

Ho L., \& Filippenko A. 1996, ApJ, 472, 600

Holtzman J.A. et al. 1992, AJ, 103, 691

Larsen S.S. 1999, A\&AS, 139, 393

Leitherer C. et al. 1999, ApJS, 123, 3

Mengel S., Lehnert M.D., Thatte N., Tacconi-Garman L.E, \& Genzel R. 2001, ApJ, 550, 280

Mihos J.C. \& Hernquist L. 1996, ApJ, 464, 641

Virgoux L. et al. 1996, A\&A, 315, L93

Whitmore B.C., Schweizer F., Leitherer C., Borne K., \& Robert C. 1993, AJ, 106,1354

Whitmore B.C., Zhang Q., Leitherer C., Fall S.M., Schweizer F., \& Miller B.W. 1999, AJ, 118, 1551

Zhang Q. \& Fall S.M. 1999, ApJ, 527, 81 